\title{
Síndrome compressiva de múltiplos nervos na dermatopolimiosite: relato de caso
}

\section{Multiple Nerve Compression Syndrome in Dermatopolymyositis: Case Report}

\author{
Marcelo José da Silva de Magalhães ${ }^{1}$ Víctor Mendes Ferreira ${ }^{2}$ \\ ${ }^{1}$ Professor, Faculdades Unidas do Norte de Minas (FUNORTE), \\ Faculdades Integradas Pitágoras de Montes Claros (FIP-Moc); \\ Neurocirurgião, Hospital Aroldo Tourinho, Montes Claros, MG, Brasil \\ 2 Acadêmico de Medicina, Faculdades Integradas Pitágoras de Montes \\ Claros, MG, Brasil

\begin{abstract}
Address for correspondence Marcelo J.S. Magalhães, MD, MSc, Rua Capelinha, 375, Antônio Pimenta, Montes Claros, MG, Brasil CEP 39402-315 (e-mail: marcelo7779@yahoo.com.br).
\end{abstract}

Arq Bras Neurocir 2016;35:85-88.

\section{Resumo \\ Palavras-Chave \\ - dermatomiosite \\ - síndrome do túnel do carpo \\ - síndrome do túnel cubital \\ - descompressão cirúrgica}

A dermatopolimiosite é miopatia inflamatória de etiologia provavelmente autoimune e comportamento heterogêneo, afetando principalmente pele e músculos e ocasionando manifestações exantemáticas características, como o eritema heliótropo e a pápula de Gottron, e fraqueza muscular proximal simétrica. A associação dessa patologia a neuropatias periféricas é pouco conhecida, podendo raramente ocorrer neuropatia múltipla. O objetivo deste artigo é relatar um caso de síndrome compressiva de múltiplos nervos em portador de dermatopolimiosite. O paciente apresentava fraqueza muscular proximal e exantema característico e foi submetido à revisão laboratorial, ressonância magnética de abdome e eletroneuromiografia, que mostraram alterações. Foi então tratado através da neurólise do nervo mediano ao nível do túnel do carpo e do nervo ulnar ao nível do túnel cubital. Trata-se de importante possibilidade terapêutica em casos como o descrito, mas estudos de maior porte sobre a descompressão simultânea dos túneis carpal e ulnar são necessários.

Dermatopolymyositis is an inflammatory myopathy - whose etiology is probably autoimmune - that has heterogeneous manifestations that occur mainly in skin and muscles and cause characteristic rash, such as heliotrope rash, Gottron's sign and symmetric proximal weakness. The association between this pathology and peripheral neuropathies is little known and multiple neuropathies rarely occur. The purpose of this article is to report a case of multiple nerve compression syndrome in a patient with dermatopolymyositis. The patient had proximal weakness and characteristic rash and underwent a laboratorial review, abdominal MRI and electromyography, which showed changes. So he was treated by neurolysis of median and ulnar nerves at carpal and cubital tunnels levels, respectively. It is an important therapeutic possibility in cases like this, but larger studies on simultaneous decompression of carpal and cubital tunnels are necessary. ISSN 0103-5355.
Copyright $(2016$ by Thieme Publicações License terms Ltda, Rio de Janeiro, Brazil
()(1) $\odot \circledast$ 


\section{Introdução}

A dermatopolimiosite (DPM) é uma miopatia inflamatória que apresenta comportamento heterogêneo, destacando-se entre as doenças reumatológicas por sua baixa frequência e grande morbidade. ${ }^{1}$ Apresenta etiologia provavelmente autoimune e afeta, a princípio, pele e músculos, ocasionando fraqueza muscular proximal simétrica e manifestações cutâneas características, como o eritema heliótropo e a pápula de Gottron. A doença constitui entidade de grande importância e demanda a intervenção terapêutica precoce, visto que responde eficientemente ao uso de agentes imunoterapêuticos. $^{2}$

A associação da DPM a neuropatias periféricas é pouco conhecida e a presença de neuropatia múltipla em paciente portador da mesma consiste em entidade rara. ${ }^{3} \mathrm{O}$ objetivo deste artigo é relatar caso de síndrome compressiva de múltiplos nervos em paciente portador de DPM, tratado através da neurólise do nervo mediano ao nível do túnel do carpo e do nervo ulnar ao nível do túnel cubital. O paciente concordou em participar da pesquisa e assinou o Termo de Consentimento Livre e Esclarecido. Não existe conflito de interesses entre os participantes do trabalho.

\section{Relato do Caso}

Paciente M. A. A. R., sexo masculino, 28 anos, com relato de fraqueza iniciada há 7 meses e acompanhada por perda de peso, alterações descamativas da pele e pneumonia. Exame clínico apresentou heliótropo em face e pápula de Gottron em mãos (- Figs. 1A-1B). Revisão laboratorial revelou aldolase e CPK elevadas. Ressonância magnética de abdome
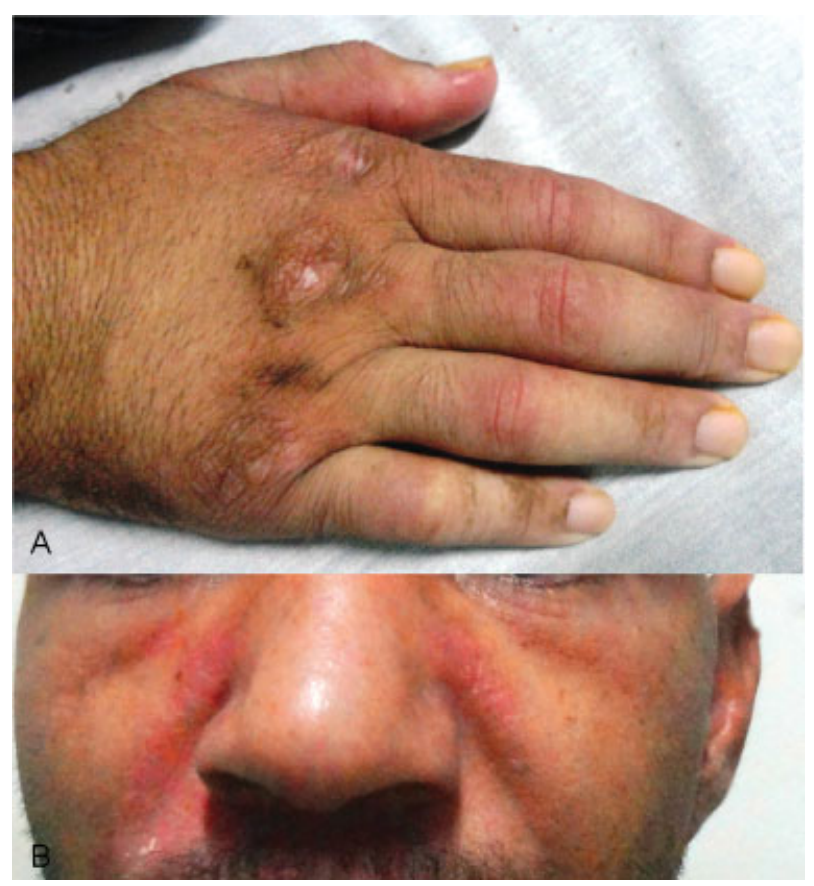

Fig. 1 (A) Pápula de Gottron em dorso da mão. (B) Sinal do heliótropo em região da face. Ambos os sinais são típicos de pacientes portadores de dermatopolimiosite. mostrou alterações na musculatura paraespinhal compatíveis com miopatia. Eletroneuromiografia (ENMG) de membros superiores e inferiores revelou quadro de síndrome do túnel do carpo (STC) bilateral, síndrome do túnel cubital (STCU), miopatia generalizada e polineuropatia sensitivomotora distal com predomínio em membros inferiores.

Exame neurológico revelou hipotrofia muscular generalizada, tetraparesia 4/5 de predomínio proximal, arreflexia de aquileu e hipostesia em padrão de bota e luva. Durante seu tratamento com prednisona, apresentou quadro neurológico compatível com STC e STCU e mais evidente em membro superior esquerdo, com comprometimento motor associado. Notou-se hipostesia acentuada em território dos Nervos ulnar e mediano, além de paresia grau $4 / 5$ dos músculos flexores do $3^{\circ}$ e $4^{\circ}$ dígitos e oponente do polegar.

Após a orientação acerca das possibilidades terapêuticas, optou-se pelo tratamento cirúrgico. 0 paciente foi submetido à descompressão simples do nervo ulnar ao nível do cotovelo e do nervo mediano ao nível do túnel do carpo. Evoluiu bem, apresentando recuperação relevante de função motora da mão esquerda, bem como redução da dor associada nos territórios dos respectivos nervos (-Figs. 2A-2B).

\section{Discussão}

Os critérios diagnósticos, definidos por Bohan \& Peter, permitem a classificação da DPM em cinco grupos: (1) juvenil, (2) primária idiopática, (3) amiopática, (4) associada a neoplasias e (5) associada a outras doenças do tecido conjuntivo. ${ }^{4}$ A suspeita clínica pode ser confirmada pelo exame das enzimas musculares séricas, sendo a mais sensível a creatinoquinase (CK), pelos achados à ENMG e por biópsia muscular. $^{5}$

As desordens sistêmicas que acometem pacientes com DPM incluem artrite reumatoide (AR), síndrome de Sjögren (SS), pneumonia etc. ${ }^{3}$ A doença ocorre mais frequentemente em indivíduos do sexo feminino, com idade média de 40 anos ao diagnóstico (na forma juvenil, de 4 a 15 anos), e demanda a intervenção terapêutica precoce, visto que responde ao uso de agentes imunossupressores. ${ }^{4,6}$

Quanto às manifestações clínicas, a doença leva a um quadro clássico de fraqueza muscular proximal simétrica e lesões cutâneas típicas, como o edema eritematoso violáceo palpebral (heliótropo) e as placas avermelhadas em superfícies extensoras das metacarpofalangianas e das interfalangianas, de distribuição simétrica (pápulas de Gottron). Além disso, alterações laboratoriais (principalmente das enzimas musculares), eletroneuromiográficas e anatomopatológicas são úteis para reforçar o diagnóstico. ${ }^{2,7}$

As anormalidades cardiovasculares, muito comuns, podem ser assintomáticas ou manifestar-se como arritmias, hipertensão arterial, pericardite e/ou miocardite. $O$ aparelho respiratório, os olhos e o aparelho urinário podem ser acometidos, levando respectivamente à doença pulmonar restritiva, vasculite retiniana e insuficiência renal aguda. Por último, podem ocorrer massas superficiais ou profundas de tamanho variável na pele (calcinose), ocasionando deformações locais, bem como perda progressiva de tecido adiposo 


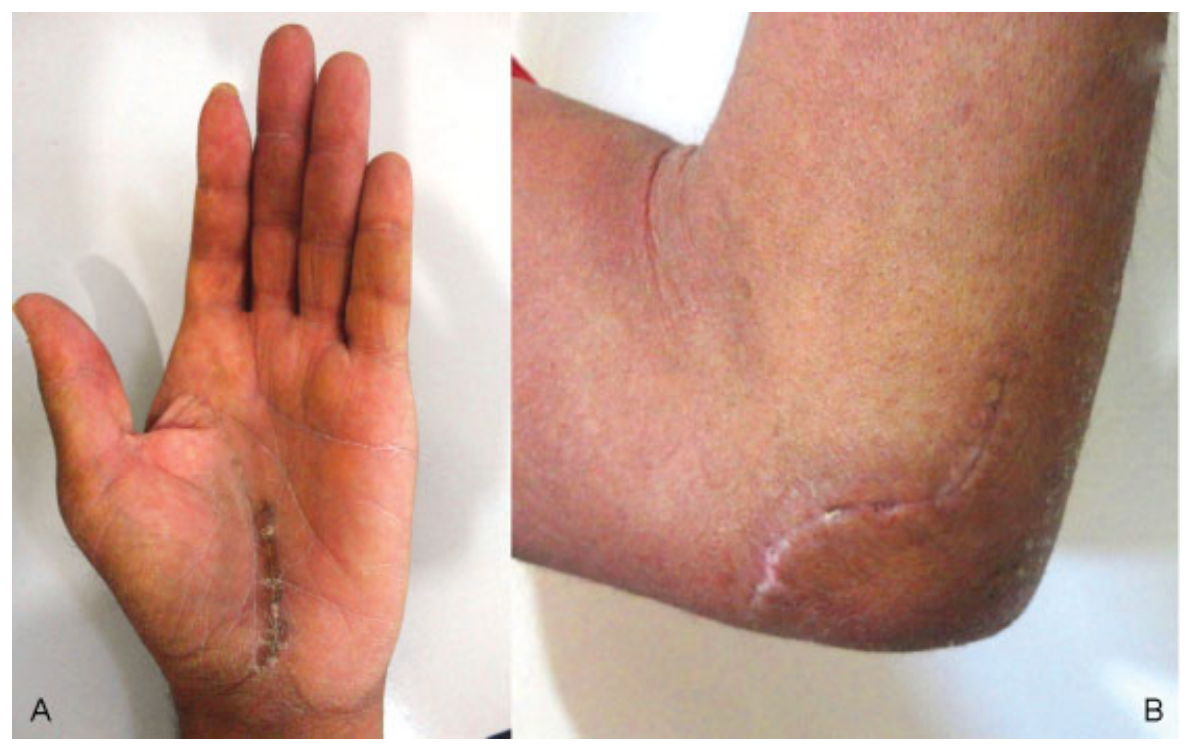

Fig. 2 (A) Cicatriz do acesso cirúrgico para síndrome do túnel do carpo, cerca de 30 dias após o tratamento. (B) Cicatriz do acesso cirúrgico para o tratamento da síndrome do túnel cubital, cerca de 30 dias após o tratamento.

subcutâneo em face e região superior do corpo (lipodistrofia). ${ }^{7}$

Em estudo recente, foram encontrados, entre 186 pacientes com DPM ou polimiosite (PM), 71 (38,2\%) achados anormais ao estudo de condução nervosa, sendo 34 (18,3\%) de neuropatia periférica. Destes, $16(8,6 \%)$ apresentavam STC, 14 (7,5\%) apresentavam polineuropatia e 4 apresentavam, respectivamente, mononeuropatia múltipla $(0,5 \%)$, neuropatia sensorial trigeminal $(0,5 \%)$, neuropatia sensorial ulnar $(0,5 \%)$ e envolvimento do plexo braquial $(0,5 \%)$. Dentre os 34 pacientes com neuropatia associada, $3(8,8 \%)$ apresentavam tumores malignos; $7,(20,6 \%)$ doenças do tecido conjuntivo; e 6, (17,6\%) diabetes. ${ }^{3}$

A STC consiste no conjunto de manifestações clínicas decorrentes de uma condição de compressão do nervo mediano no ponto em que este atravessa o punho. Trata-se da neuropatia compressiva mais comum dos membros superiores, cujas queixas mais comuns são de parestesias constantes ou intermitentes e dormência no território do referido nervo, associadas a dor (às vezes, chegando a acordar o paciente durante a noite). Em casos mais graves, pode ocorrer atrofia da musculatura tenar e fraqueza na oponência do polegar. ${ }^{8,9}$ A síndrome pode estar relacionada a fatores ocupacionais, como o ato repetido de flexão, pronação e supinação do punho, e fatores não ocupacionais, que incluem ocorrência prévia de fratura de punho, artrite reumatoide, osteoartrite de punho ou carpo, obesidade, diabetes e o uso de insulina, sulfonilureias, metformina e tiroxina, entre outros. $^{10,11}$

A compressão do nervo ulnar ao nível do cotovelo é também frequente na prática clínica, constituindo, depois da STC, a neuropatia compressiva mais comum dos membros superiores. ${ }^{12}$ A STCU é possibilitada pela complexidade do trajeto do nervo ulnar no cotovelo e apresenta forte associação com as variações anatômicas do ligamento retinacular cubital, entre outras alterações congênitas e metabólicas, além de sequelas de trauma do cotovelo, osteoartrose, estresse crônico em valgo etc. ${ }^{13,14}$ Os pacientes geralmente apresentam parestesias no território de inervação do nervo ulnar e atrofia da musculatura intrínseca da mão. A extensão da disfunção neural foi estratificada por McGowan em três graus: apenas alterações sensitivas (grau I), fraqueza muscular (grau II), e paresia e atrofia muscular (grau III). ${ }^{14}$

O tratamento conservador da STC pode ser utilizado nos pacientes com as seguintes características: doença com menos de um ano de evolução, ausência de déficit motor ou atrofia tenar, ausência de sinais de desnervação à ENMG. ${ }^{15}$

Quanto ao tratamento conservador da STC, a injeção local de esteroide não tem sido muito aceita como opção, por aumentar o risco de lesão direta do nervo pela infiltração intraneural e apresentar efeito transitório. É utilizada nos casos em que a cirurgia não pode ser realizada. ${ }^{15}$ Outra opção de tratamento conservador é a imobilização do membro acometido através de tala. ${ }^{15}$ Durante o seu uso, o paciente não realiza a flexão do punho, reduzindo a compressão do nervo mediano ao longo da noite e, por conseguinte, reduzindo as parestesias. $\mathrm{O}$ uso de vitamina $\mathrm{B} 6$ não tem mostrado resultados satisfatórios. ${ }^{15}$

Nos pacientes com sinais de hipotrofia muscular ou hipostesia permanente, a liberação do nervo mediano como tratamento da STC está indicada. ${ }^{15}$ Entre as técnicas cirúrgicas disponíveis para o tratamento da STC, encontra-se a cirurgia aberta desenvolvida por Phalen na década de 1950. Possui inúmeras variações quanto ao tipo de incisão e visa à secção do ligamento transverso do carpo liberando o nervo mediano da compressão ao nível do punho. Esta técnica apresenta baixos índices de complicação. ${ }^{15}$

A técnica endoscópica desenvolvida nos anos 1980 apresenta duas variantes: a técnica monoportal, com uma incisão 
realizada ao nível do punho, e a técnica biportal, com uma incisão realizada ao nível do punho e outra na região palmar. Apesar de ser uma técnica menos invasiva, apresenta como dificuldade a curva de aprendizado mais longa do cirurgião, quando comparada à técnica clássica. Os resultados são similares entre as técnicas endoscópica e clássica, mas com o retorno do paciente mais rapidamente ao trabalho na técnica endoscópica. ${ }^{15}$

O tratamento conservador da STCU está indicado para os pacientes com sintomas intermitentes, neuropatia leve ou moderada, e consiste em evitar os movimentos de flexão forçada do cotovelo por meio de mudança de hábito e/ou imobilização e uso de anti-inflamatórios não hormonais. Nos pacientes com déficits neurológicos e alterações de inervação na ENMG, a cirurgia para o tratamento da STCU está indicada. Existem duas técnicas distintas para o tratamento da neuropatia do nervo ulnar ao nível do cotovelo: a descompressão simples, na qual o nervo ulnar é exposto em todo o seu trajeto na região do cotovelo, e uma segunda técnica, na qual, adicionalmente, o nervo é transposto à região anterior da fossa cubital e posicionado no tecido celular subcutâneo ou sob a musculatura flexora-pronadora. ${ }^{16}$

Atualmente, as técnicas de liberação endoscópica do túnel carpal e do túnel cubital são consideradas seguras e eficazes para o tratamento isolado da STC e da STCU, respectivamente. ${ }^{19}$ Estudo recente, que avaliou os resultados da técnica de liberação endoscópica simultânea dos túneis carpal e ulnar em 17 pacientes, demonstrou eficácia e segurança, sendo os resultados e complicações pós-operatórios comparáveis àqueles inerentes ao uso isolado das duas técnicas. No referido estudo, 82 e 100\% dos pacientes apresentaram resolução completa da dor e dos sintomas de nervo mediano, respectivamente. $^{17}$

O tratamento cirúrgico leva a alívio significativo dos sintomas, bem como previne estágios mais avançados de disfunção. As complicações da liberação endoscópica e da cirurgia aberta incluem lesões de estruturas nervosas, lesões vasculares, lesões de tendões, infecções locais e deiscência de ferida cirúrgica. ${ }^{18}$

\section{Conclusão}

O diagnóstico de neuropatias compressivas deve sempre ser aventado em pacientes portadores de DPM que apresentem queixas sensoriais e/ou motoras - especialmente nos membros, sem outro fator causador prontamente evidente. A técnica de descompressão clássica dos nervos ulnar e mediano pode ser uma opção de tratamento para estes pacientes. Estudos envolvendo uma casuística maior se fazem necessários para avaliar a descompressão simultânea do túnel carpal e do túnel cubital em pacientes portadores da doença reumatológica em questão.

\section{Referências}

1 Marchiori PE, Hirata MTA, Scaff M, et al. Dermatopolymyositis: evaluation of 63 patients. Arq Neuropsiquiatr 1987;45(2): 137-142

2 Pereira FC, Rezende CM, Barbosa SVC, Wisniewski ED, Uyeda H, Cristovam MAS. Dermatopolimiosite juvenil: relato de caso. Rev Med Resid 2011;13(2):1-5

3 Wang Y, Cui LY, Chen L, et al. Nerve conduction studies in patients with dermatomyositis or polymyositis. Chin Med J (Engl) 2010; 123(5):523-526

4 Ortigosa LCM, Reis VMS. Dermatomiosite. An Bras Dermatol 2008;83(3):247-259

5 Dalakas MC. Polimiosite, dermatomiosite e miosite com corpúsculos de inclusão. In: Fauci AS, Braunwald E, Kasper DL, Hauser SL, Longo DL, Jameson JL. et al. Harrison medicina interna. 17th ed. Rio de Janeiro: McGraw-Hill Interamericana do Brasil; 2008. p. 2696-703.

6 Dalakas MC, Hohlfeld R. Polymyositis and dermatomyositis. Lancet 2003;362(9388):971-982

7 Oliveira SKF. Polimiosite e dermatomiosite na infância. In: Carvalho MAP, Lanna CCD, Bértolo MB. Reumatologia: diagnóstico e tratamento. 3rd ed. Rio de Janeiro: Guanabara Koogan; 2011. p. 523-30.

8 Alves MPT. Liberação do canal do carpo por mini-incisão transversa. Acta Ortop Bras. 2011;19(6):362-367

9 Carvalho KMD, Soriano EP, Carvalho MVD, Mendoza CC, Vidal HG, Araújo ABVL. Nível de evidência e grau de recomendação dos artigos sobre a acurácia diagnóstica da ultrassonografia na síndrome do túnel do carpo. Radiol Bras 2011;44(2):85-89

10 Tanaka S, Wild DK, Cameron LL, Freund E. Association of occupational and non-occupational risk factors with the prevalence of self-reported carpal tunnel syndrome in a national survey of the working population. Am J Ind Med 1997;32(5):550-556

11 Geoghegan JM, Clark DI, Bainbridge LC, Smith C, Hubbard R. Risk factors in carpal tunnel syndrome. J Hand Surg [Br] 2004;29(4): 315-320

12 Bartels RHMA, Menovsky T, Van Overbeeke JJ, Verhagen WIM. Surgical management of ulnar nerve compression at the elbow: an analysis of the literature. J Neurosurg 1998;89(5):722-727

13 Viveiros MEM, Matsumoto MH, Bastos TB, Moraes MJS, Uehara A, Pereira $\mathrm{H}$. Tratamento da síndrome do túnel ulnar pela técnica da epicondilectomia parcial medial do cotovelo. Rev Bras Ortop 2008;43(11-12):490-496

14 Lima S, Correia JF, Martins RM, Alves JM, Palheiras J, Sousa C. Tratamento da síndrome do túnel cubital pela técnica de transposição anterior subcutânea: será este método seguro e eficaz? Rev Bras Ortop 2012;47(6):748-753

15 Flores LP. Compressão do nervo mediano no punho (síndrome do túnel do carpo). In: Siqueira MG, Martins RS. Síndromes compressivas de nervos periféricos. 1st ed. Rio de Janeiro: Di Livros; 2009. p. 29-48.

16 Carvalho GA. Compressão do nervo ulnar no cotovelo (síndrome do túnel cubital). In: Siqueira MG, Martins RS. Síndromes compressivas de nervos periféricos. 1st ed. Rio de Janeiro: Di Livros; 2009. p. 49-58.

17 Cross D, Matullo KS. Concomitant endoscopic carpal and cubital tunnel release: safety and efficacy. Hand (NY) 2014;9(1):43-47

18 Palmer BA, Hughes TB. Cubital tunnel syndrome. J Hand Surg Am 2010;35(1):153-163

19 Palmer AK, Toivonen DA. Complications of endoscopic and open carpal tunnel release. J Hand Surg Am 1999;24(3):561-565 